\title{
ЭЛЕКТРОХИМИЧЕСКАЯ ОБРАБОТКА ОБЛУЧЕННОГО ГРАФИТА УРАН-ГРАФИТОВЫХ ЯДЕРНЫХ РЕАКТОРОВ
}

Павлюк Александр Олегович',

seversknet@rambler.ru

Беспала Евгений Владимирович',

bespala_evgeny@mail.ru

Котляревский Сергей Геннадьевич',

skotkyarevkiy@rambler.ru

Волкова Анна Генриховна²,

anna.agv@yandex.ru

\section{Захарова Елена Васильевна²,}

zevchem@rambler.ru

\author{
АО «Опытно-демонстрационный центр вывода из эксплуатации уран-графитовых ядерных реакторов», \\ Россия, 636000, г. Северск, ул. Автодорога 13, 179а. \\ 2 Институт физической химии и электрохимии им. А.Н. Фрумкина Российской академии наук, \\ Россия, 119071, г. Москва, пр. Ленинский, 31, корп. 4.
}

Актуальность исследования обусловлена необходимостью разработки эффективных методов дезактивации облученного графита уран-графитовых ядерных реакторов с целью снижения потенциальной опасности для дальнейшего захоронения. Цель: разработка метода электрохимической дезактивации графитовых радиоактивных отходов в химически агрессивных средах и определение оптимальных параметров ведения процесса.

Методы: экспериментальные исследования процесса электрохимической обработки облученного графита в различных электролитах и при различных условиях.

Результаты. Показана принципиальная возможность электрохимической обработки графитовых радиоактивных отходов. Представлена конструкция электролизера для проведения процесса обработки и описана методика проведения эксперимента. Проанализированы электродные процессы и определены факторы, влияющие на эффективность электрохимической дезактивации. Представлены зависимости скорости разрушения графитовых анодов от плотности тока и различных концентраций кислот. Определено, что наибольшая скорость разрушения электродов наблюдается в концентрированной азотной кислоте. Экспериментально были получены зависимости, отражающие степень очистки графитовых РАО по радионуклидам ${ }^{60} \mathrm{Co},{ }^{134+137} \mathrm{Cs},{ }^{154} \mathrm{Eu}$, ${ }^{241} \mathrm{Am}$ в зависимости от состава электролита. Выявлено, что максимальная степень дезактивации облученного ядерного графита при плотности тока 0,01 A/CM² и температуре электролита $(16-20){ }^{\circ} \mathrm{C}$ достигается в азотной кислоте с добавлением 0,2 M NaF. Проведен сравнительный анализ результатов экспериментов химической и электрохимической обработки графитовых радиоактивных отходов. Показано, что электрохимическая дезактивация графита позволяет снизить активность графита по ${ }^{60}$ Со в 2-10 раз и по ${ }^{137} \mathrm{Cs}$ в 7-100 раз. При этом в процессе дезактивации не образуется газообразных продуктов реакции, которые необходимо улавливать, создавая ещё большее количество вторичных радиоактивных отходов.

\section{Ключевые слова:}

Реакторный графит, электрохимическая обработка, радионуклид, уран-графитовый реактор, дезактивация

\section{Введение}

В настоящее время в мире накоплено более 250000 т графитовых радиоактивных отходов (РА0). Ранее выявлено, что активность таких РАО определяется активационными радионуклидами $\left({ }^{3} \mathrm{H},{ }^{14} \mathrm{C},{ }^{55} \mathrm{Fe},{ }^{60} \mathrm{Co}\right.$ и др. $)$, продуктами деления $\left({ }^{90} \mathrm{Sr}\right.$, ${ }^{106} \mathrm{Ru},{ }^{125} \mathrm{Sb},{ }^{133} \mathrm{Ba},{ }^{137} \mathrm{Cs},{ }^{154} \mathrm{Eu},{ }^{155} \mathrm{Eu}$ и др.) и трансурановыми элементами $\left({ }^{241} \mathrm{Am},{ }^{243} \mathrm{Am},{ }^{244} \mathrm{Cm}\right.$ и др.) [1-5]. Для решения проблемы графитовых РАО в РФ рассматриваются варианты их размещения в приповерхностных пунктах захоронения радиоактивных отходов (ППЗРО) и в пункте глубинного захоронения радиоактивных отходов (ПГЗРО) в Нижне-Канском массиве. В соответствии с ФЗ Российской Федерации «Об обращении с радиоактив- ными отходами...» (от 11.07.2011 № 190-ФЗ) и Постановлением Правительства Российской Федерации № 1069 от 19.10.2012 «0 критериях отнесения...» удаляемые графитовые РАО, отнесенные к 3 или 4 классу, могут быть захоронены в ППЗРО. При отнесении облученного графита к 1 и 2 классу удаляемых PAO (энергетические реакторы типа АМБ и др.) возникает необходимость его захоронения в ПГЗРО [6]. Графит промышленных уран-графитовых реакторов (ПУГР), являющийся ядерным наследием, может быть отнесен к особым (не удаляемым) РА0, что позволяет реализовать вариант «захоронение на месте» $[7,8]$.

Стоимость обращения с облученным ядерным графитом существенно зависит от способа его захо- 
ронения, который определяется классом РАО. Граница между 2 и 3 классами РАО по величине бетаизлучающих радионуклидов с периодом полураспада более 31 года составляет $10^{4}$ Бк/г и трансурановых радионуклидов $10^{2}$ Бк/г. Изменение класса графитовых PAO со 2-го на 3-й путем снижения активности графита позволит уйти от наиболее затратного и технически труднореализуемого способа захоронения в глубоких геологических формациях и реализовать более предпочтительный с точки зрения вопросов экономики и технологически более простой способ приповерхностного захоронения при обеспечении необходимого уровня безопасности для населения. Поэтому существует экономическая целесообразность в поиске путей изменения класса графитовых отходов путем переработки или дезактивации.

В настоящее время разрабатываются и тестируются различные методы воздействия на облученный реакторный графит, к которым можно отнести: прямое сжигание в окислительной среде $[9-11]$, пиролиз $[12,13]$, плазменную обработку $[14,15]$ и др. При этом применение таких методов для очистки графита приводит к образованию радиоактивных газообразных продуктов, что создает сложности при последующем обращении с газообразными вторичными РАО. В связи с этим в работе был апробирован метод электрохимической дезактивации, исключающий перевод облученного графита в газовую фазу.

В атомной промышленности для дезактивации металлических поверхностей используется электролитическая очистка $[16,17]$. В этом случае происходит концентрирование загрязнителей в растворе электролита, что исключает образование опасных газообразных продуктов реакции. Ранее предпринимались попытки адаптировать известные методы электрохимической дезактивации к проблеме обращения с облученным ядерным графитом [18], но исследования на реальных образцах облученного графита не проводились.

Целью настоящей работы является исследование процесса электрохимической обработки облученного ядерного графита уран-графитового реактора. Для оценки перспективности метода на настоящем этапе выполнялось определение степени дезактивации по относительно легко измеряемым радионуклидам $\left({ }^{60} \mathrm{Co},{ }^{134} \mathrm{Cs},{ }^{137} \mathrm{Cs},{ }^{154} \mathrm{Eu},{ }^{241} \mathrm{Am}\right)$, которые существенно влияют на общую активность графита и в значительной мере сосредоточены в приповерхностном слое графита. Как показали исследования, в этом слое графита также могут находиться более высокие концентрации ${ }^{14} \mathrm{C}$ и ${ }^{36} \mathrm{Cl}$ [19]. Причем ${ }^{36} \mathrm{Cl}$ может оказывать существенное влияние на обоснование безопасности пункта захоронения РАО [20-23].

Удаление радионуклидов, сосредоточенных на поверхности, также может привести к снижению потенциальной опасности графитовых радиоактивных отходов [24-26].

\section{Электродные процессы при обработке}

\section{облученного графита}

Дезактивация облученного ядерного графита методом электролиза заключается, в первую очередь, в удалении загрязнённого поверхностного слоя. Это обусловлено поверхностным загрязнением образцов, включая поверхность пор. Известно, что разрушение графита происходит при использовании его в качестве анодов в электрохимической промышленности. При электролизе графит разрушается не в результате электрохимического процесса, например процесса окисления ионов хлора, а вследствие выделения кислорода на аноде по реакции (1), потенциал которой равен 1,23 В относительно нормального водородного электрода [27]:

$$
\mathrm{H}_{2} \mathrm{O}-2 \mathrm{e}^{-}=0,5 \mathrm{O}_{2}+2 \mathrm{H}^{+} \text {. }
$$

Износ анодов возникает не только как следствие окисления углерода, но и из-за осыпания графитовой массы после того, как нарушается структура поверхностных слоев. Процесс разрушения анодов развивается главным образом в глубине пор. Проникновению электролита в глубь пор способствует увеличение смачиваемости поверхности при возрастании анодного потенциала [28]. В порах из-за развитой поверхности и низкой плотности тока создаются благоприятные условия для выделения кислорода, и в результате факторы, влияющие на интенсивность выделения кислорода при электролизе, способствуют разрушению графитового анода.

При исследовании возможности интенсификации процессов электрохимического получения хлора и хлоратов было обнаружено [29], что существует некоторый предельный анодный потенциал, названный «критическим», выше которого наблюдается резкое возрастание износа графитовых анодов. Это явление целесообразно использовать для дезактивации облученного ядерного графита.

Рассматривая механизм разрушения графитовых анодов, следует учитывать, что увеличение износа в закритической области обусловлено сочетанием двух факторов: достижением критического потенциала, выше которого энергетическое состояние анодной поверхности создает условия для образования оксидных фаз, и наличием возможности разряда кислородосодержащих анионов или воды, являющихся источником кислорода, необходимого для окисления анода. При отсутствии хотя бы одного из этих условий увеличение износа не наступает.

В работе [30] описано повышение износа графита при проведении электролиза при температурах выше $40{ }^{\circ} \mathrm{C}$.

Электрохимическая дезактивация при поляризации поверхности в растворах электрическим током является в настоящее время наиболее эффективным способом очистки от радиоактивных изотопов, позволяющим проводить очистку в растворах кислот и их солей при нормальных температу- 
рах. Эффективность такой обработки основана на изменении в процессе поляризации валентности поверхностных атомов и ионов, за счет чего происходит образование на поверхности новых соединений с более высокой реакционной способностью, обладающих повышенной растворимостью в дезактивирующих растворах.

Наиболее подходящими электролитами для максимального выделения на электродах кислорода и водорода при электролизе являются кислородосодержащие неорганические кислоты, такие как: азотная, хлорная, серная и фосфорная.

Для определения оптимальных параметров процесса дезактивации облученного ядерного графита была разработана экспериментальная установка (рис. 1). Расходуемые электроды - 1, выполненные из облученного графита уран-графитового реактора (рис. 2), размещались в различных секциях электролизера - 2, который с торцов был снабжен патрубком подачи - 3 и патрубком отвода -4 дезактивирующего раствора - 5 (электролита). При этом катод и анод в электролизере -2 чередовались по всей его длине, а секции были разделены ионообменными мембранами - 6. Расходуемые электроды - 1 через изоляторы - 7, расположенные в массивной крышке - 8 электролизера - 2 посредством проводов - 9 соединялись с источником тока - 10

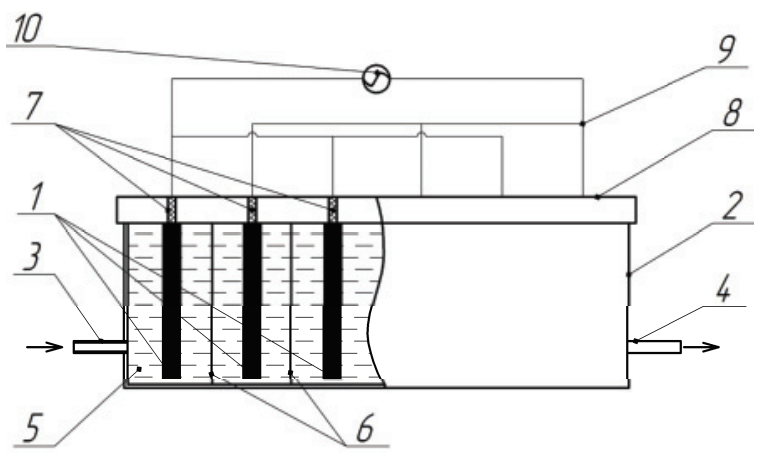

Pис. 1. Схема экспериментальной установки для электролитической обработки облученного графита

Fig. 1. Scheme of the experimental installation for electrochemical treatment of irradiated graphite

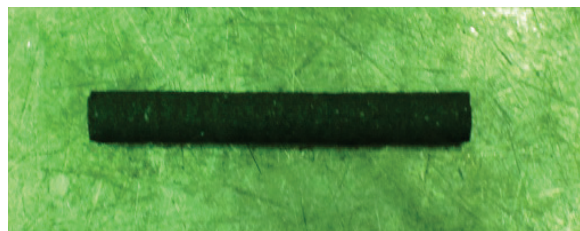

Pис. 2. Внешний вид электрода из облученного графита

Fig. 2. Appearance of the electrode from irradiated graphite

Как показали эксперименты (табл. 1), скорость разрушения графита в $8 \mathrm{M}$ серной и фосфорной кислот оказалась наименьшей и равной 0,12 и 0,06 г/( $\mathrm{cm}^{2}$.ч) соответственно. Самые высокие скорости разрушения графита в аналогичных условиях были получены в хлорной кислоте
0,72 г/( $\left.\mathrm{cm}^{2} \cdot ч\right)$. Однако в процессе окисления графита хлорная кислота разлагалась до ионов хлора, которые являются коррозионно-агрессивными по отношению к стали, являющейся конструкционным материалом для изготовления электролизных ванн. Таким образом, только азотная кислота являлась наиболее подходящим электролитом для разрушения графита при его анодной поляризации. При достаточно высокой скорости разрушения графита кислота коррозионно не агрессивна по отношению к высоколегированной стали марки 02Х8Н22С6 (ЭП794). Способы очистки поверхности данной марки стали от радионуклидов с использованием растворов азотной кислоты широко известны и применяются в промышленности [21]. Скорости разрушения графита в 4-8 и 14,3 М растворах азотной кислоты достаточно близкие и составляют, соответственно, 0,26-0,32 и 0,42 г/( $\left.\mathrm{cm}^{2} \cdot ч\right)$ при плотности тока $0,2 \mathrm{~A} / \mathrm{cm}^{2}$.

таблица 1. Скорость разрушения графита $\left(г /\left(\mathrm{CM}^{2} \cdot 4\right)\right)$ в растворах кислот в процессе электролиза при плотности анодного тока $0,2 \mathrm{~A} / \mathrm{CM}^{2}$

Table 1. Rate of graphite destruction $\left(\mathrm{g} /\left(\mathrm{cm}^{2} \cdot \mathrm{h}\right)\right)$ in acid solutions during electrolysis at an anodic current density of $0,2 \mathrm{~A} / \mathrm{cm}^{2}$

\begin{tabular}{|c|c|c|c|c|}
\hline \multirow{2}{*}{$\begin{array}{c}\text { Кислота } \\
\text { Acid }\end{array}$} & \multicolumn{4}{|c|}{ Концентрация (М)/Concentration (M) } \\
\cline { 2 - 5 } & 2 & 4 & 8 & 14,3 \\
\hline $\mathrm{HNO}_{3}$ & 0,09 & 0,26 & 0,32 & 0,42 \\
\hline $\mathrm{HClO}_{4}$ & 0,23 & 0,7 & 0,72 & - \\
\hline $\mathrm{H}_{2} \mathrm{SO}_{4}$ & - & 0,05 & 0,12 & - \\
\hline $\mathrm{H}_{3} \mathrm{PO}_{4}$ & - & - & 0,06 & - \\
\hline
\end{tabular}

При увеличении плотности тока от 0,15 до $0,25 \mathrm{~A} / \mathrm{cm}^{2}$ скорость разрушения графита сначала увеличивалась (максимум при плотности тока $0,25 \mathrm{~A} / \mathrm{cm}^{2}$ ), а затем уменьшалась (рис. 3). Начиная с $0,15 \mathrm{~A} / \mathrm{cm}^{2}$ и выше, режим электролиза становился неустойчивым из-за бурного выделения газа на электродах.

При снижении плотности тока до 0,2 A/ $\mathrm{cm}^{2}$ или концентрации азотной кислоты ниже $4 \mathrm{M}$ наблюдалось уменьшение скорости разрушения графита.

Введение в раствор кислот дополнительных реагентов, способных разлагаться на электродах интенсивнее, чем ионы воды, то есть имеющих менее положительный потенциал разрядов ионов (в случае анодной поляризации), чем потенциал выделения кислорода, приводило к расходованию подаваемого анодного тока на разрушение этих реагентов. При этом изменялась скорость разрушения графита: от нуля до некоторой величины, не превышающей величину скорости разрушения графита в растворе кислоты, не содержащем посторонних реагентов.

Результаты экспериментов, приведенные в табл. 2, показали, что введение щавелевой кислоты $\mathrm{H}_{2} \mathrm{C}_{2} \mathrm{O}_{4} 0,22 \mathrm{M}$ в $4 \mathrm{M} \mathrm{HNO}_{3}$ приводит к уменьшению скорости разрушения графита с 0,26 до 

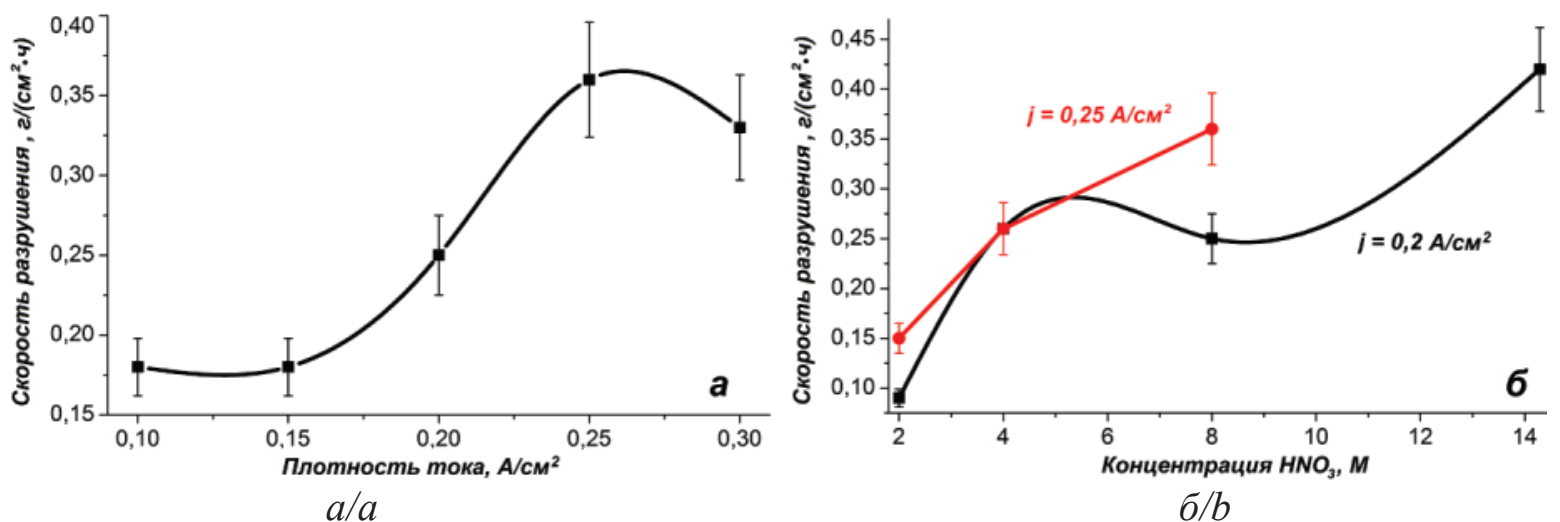

$\sigma / b$

Рис. 3. Скорость разрушения облученного графита в азотной кислоте в зависимости от: а) плотности тока в $8 \mathrm{M} \mathrm{HNO}_{3}$; б) концентрации $\mathrm{HNO}_{3}$ при различных плотностях тока

Fig. 3. Rate of destruction of irradiated graphite in nitric acid, depending on: a) current density in $8 \mathrm{M} \mathrm{HNO}_{3}$; b) concentrations of $\mathrm{HNO}_{3}$ at different current densities

0,19 г/( $\mathrm{cm}^{2} \cdot$-ч) из-за ее разложения на аноде с выделением диоксида углерода по реакции (2):

$$
\mathrm{H}_{2} \mathrm{C}_{2} \mathrm{O}_{4} \rightarrow 2 \mathrm{CO}_{2}+2 \mathrm{H}^{+}+2 \mathrm{e}^{-} \text {. }
$$

Нормальный окислительно-восстановительный потенциал этой реакции, равный $0,49 \mathrm{~B}$, имеет более отрицательное значение, чем потенциал выделения кислорода из воды, равный 1,229 В. В щавелевой кислоте $(0,89 \mathrm{M})$ протекала только анодная реакция ее разрушения с выделением диоксида углерода. Вследствие отсутствия на аноде выделения кислорода разрушение графита происходило относительно медленно.

Таблица 2. СКорость разрушения графита $\left(\left\ulcorner/\left(\mathrm{CM}^{2} .4\right)\right)\right.$ в азотной кислоте в присутствии различных добавок при электролитической обработке и разных плотностях тока

Table 2. Rate of graphite destruction $\left(\mathrm{g} /\left(\mathrm{cm}^{2} \cdot \mathrm{h}\right)\right)$ in nitric acid in the presence of various additives during electrolytic treatment and different current densities

\begin{tabular}{|c|c|c|}
\hline \multirow{2}{*}{$\begin{array}{c}\text { Состав электролита } \\
\text { Electrolyte composition }\end{array}$} & \multicolumn{2}{|c|}{$\begin{array}{c}\text { Плотность тока }\left(\mathrm{A} / \mathrm{cm}^{2}\right) \\
\text { Current Density }\left(\mathrm{A} / \mathrm{cm}^{2}\right)\end{array}$} \\
\cline { 2 - 3 } & 0,15 & 0,20 \\
\hline $8 \mathrm{M} \mathrm{HNO}_{3}+0,2 \mathrm{M} \mathrm{NaF}$ & 0,19 & 0,26 \\
\hline $4 \mathrm{M} \mathrm{HNO}_{3}+0,22 \mathrm{M} \mathrm{H}_{2} \mathrm{C}_{2} \mathrm{O}_{4}$ & - & 0,19 \\
\hline $0,89 \mathrm{M} \mathrm{H}_{2} \mathrm{C}_{2} \mathrm{O}_{4}$ & 0 & - \\
\hline $4 \mathrm{M} \mathrm{HNO}_{3}+2,94 \mathrm{M} \mathrm{H}_{2} \mathrm{O}_{2}$ & - & 0 \\
\hline $8 \mathrm{M} \mathrm{HNO}_{3}+0,025 \mathrm{M} \mathrm{H}_{3} \mathrm{PO}_{4}$ & - & 0,23 \\
\hline $8 \mathrm{M} \mathrm{HNO}_{3}+0,05 \mathrm{M} \mathrm{H}_{2} \mathrm{SO}_{4}$ & - & 0,22 \\
\hline $8 \mathrm{M} \mathrm{HNO}_{3}+0,1 \mathrm{M} \mathrm{H}_{2} \mathrm{SO}_{4}$ & - & 0,24 \\
\hline $\mathrm{HNO}_{3}: \mathrm{H}_{2} \mathrm{SO}_{4}=1: 1$ & - & 0,24 \\
\hline $\mathrm{HNO}_{3}: \mathrm{HClO}_{4}=3: 1$ & - & 0,25 \\
\hline $\mathrm{HNO}_{3}: \mathrm{HClO}_{4}=1: 1$ & - & 0,7 \\
\hline
\end{tabular}

В случае, когда в раствор $4 \mathrm{M}$ азотной кислоты вводили 2,94 М пероксида водорода $\mathrm{H}_{2} \mathrm{O}_{2}$, нормальный окислительно-восстановительный потенциал которой равен $0,68 \mathrm{~B}$, скорость разрушения графита падала до нулевых значений. Однако по мере разложения пероксида водорода в процессе электролиза (примерно через 30 минут обработки) вновь наблюдалось разрушение графита со скоростью 0,12 г $/\left(\mathrm{cm}^{2} \cdot\right.$ ч). При повторном добавлении $\mathrm{H}_{2} \mathrm{O}_{2}$ разрушение графита прекращалось.

Введение в кислоты реагентов, не разрушающихся при потенциалах выделения кислорода $(1,65-1,69)$ В (с учетом перенапряжения), например, как ионы фтора, нормальный потенциал выделения которого в виде газообразного фтора равен $2,87 \mathrm{~B}$, практически не приводило к изменению скорости разрушения графита. Например, при проведении электролиза в $8 \mathrm{M} \mathrm{HNO}_{3}$ с добавкой $0,2 \mathrm{M}$ фторида натрия $\mathrm{NaF}$ и без нее скорость разрушения графита оставалась на уровне $(0,26-0,32)$ г/( $\left.\mathrm{cm}^{2} . ч\right)$ (табл. 1$)$.

\section{Экспериментальное исследование процесса дезактивации облученного графита электрохимическим методом}

Поверхностная дезактивация ядерного графита и дезактивация его с одновременным разрушением электрода проводилась на образцах, изготовленных из графитовых блоков и втулок уран-графитового ядерного реактора с водяным охлаждением. Образцы изготовлялись из графита, находящегося на поверхности (таблетки 1Б, 2, 3, 7, 8, 9) и внутри (таблетки 4,5 и 6 ) графитовых элементов. При этом образцы, вырезанные из блоков, имели индекс «Б», все остальные - без индекса. Начальные уровни загрязнения приведены в табл. 3 .

Загрязненность графита, как следует из данных табл. 3, определялась в основном изотопами ${ }^{60} \mathrm{Co}$ и ${ }^{137} \mathrm{Cs}$. Радионуклид ${ }^{60} \mathrm{Co}$ в графите образовывался в основном за счет активации микропримесей, находящихся в объеме графита. Кроме того, кобальт, как и цезий, накапливался на поверхностях втулок после протечек теплоносителя в активную зону, с последующей диффузией нуклидов в объем графита, что подтверждается наличием объемного загрязнения образцов цезием. В исследованных образцах втулок практически не было зафиксировано присутствие $\alpha$-излучателей. 
Таблица 3. Исходная активность образцов облученного графита

Table 3. Initial activity of samples of irradiated graphite

\begin{tabular}{|c|c|c|c|c|c|c|}
\hline \multirow{3}{*}{$\begin{array}{l}\text { Образец } \\
\text { Specimen }\end{array}$} & \multicolumn{6}{|c|}{$\begin{array}{l}\text { Начальная загрязненность, Бк/г } \\
\text { Initial activity concentration, Bq/g }\end{array}$} \\
\hline & \multirow{2}{*}{$\begin{array}{l}\text { Общая } \\
\text { Total }\end{array}$} & \multicolumn{5}{|c|}{ По радионуклидам/Radionuclides } \\
\hline & & ${ }^{60} \mathrm{Co}$ & ${ }^{134} \mathrm{Cs}$ & ${ }^{187} \mathrm{Cs}$ & ${ }^{154} \mathrm{Eu}$ & ${ }^{241} \mathrm{Am}$ \\
\hline 15 & 13860 & 12434 & 267 & 1117 & 21 & 21 \\
\hline 2 & 14398 & 3475 & 273 & 10650 & - & - \\
\hline 3 & 12606 & 4389 & 187 & 8030 & - & - \\
\hline 4 & 1079 & 580 & - & 499 & - & - \\
\hline 5 & 1666 & 1051 & - & 315 & - & - \\
\hline 6 & 2016 & 1719 & - & 296 & - & - \\
\hline 7 & 1146 & 797 & 30 & 319 & - & - \\
\hline 8 & 2186 & 1712 & 15 & 459 & - & - \\
\hline 9 & 1698 & 1319 & 3 & 376 & - & - \\
\hline
\end{tabular}

Электрохимическая обработка проводилась при анодной поляризации графита в азотнокислых растворах. При этом плотность тока составляла порядка $0,01 \mathrm{~A} / \mathrm{cm}^{2}$ при поверхностной дезактивации и $0,2 \mathrm{~A} / \mathrm{cm}^{2}$ - при дезактивации с одновременным разрушением графита до порошкообразного состояния. Данные по электрохимической дезактивации графита при плотности тока $0,01 \mathrm{~A} / \mathrm{cm}^{2}$ приведены в табл. 4. В табл. 5 представлены данные, полученные при химической дезактивации графита из втулок, отобранных вдали от аварийных ячеек.

Таблица 4. Результаты электрохимической дезактивации образцов облученного графита 1Б, 8 и 6 при $j=0,01 \mathrm{~A} / \mathrm{CM}^{2},(16-20)^{\circ} \mathrm{C}$, продолжительность процecca 24

Table 4. Results of electrochemical decontamination of samples of irradiated graphite 15,8 and 6 at $j=0,01 \mathrm{~A} / \mathrm{cm}^{2},(16-20){ }^{\circ} \mathrm{C}$, the duration of the process is $2 \mathrm{~h}$

\begin{tabular}{|c|c|c|c|c|c|c|c|}
\hline \multirow{2}{*}{ 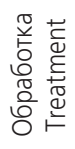 } & \multirow[t]{2}{*}{$\begin{array}{l}\text { Состав раствора } \\
\text { Solution structure }\end{array}$} & \multirow[t]{2}{*}{$\begin{array}{l}\text { Oбp } \\
\text { Spe. }\end{array}$} & \multicolumn{5}{|c|}{$\begin{array}{c}\text { Остаточная загрязнен- } \\
\text { ность, \% } \\
\text { Residual contamination, \% }\end{array}$} \\
\hline & & & ${ }^{60} \mathrm{Co}$ & ${ }^{134} \mathrm{CS}$ & ${ }^{137} \mathrm{Cs}$ & ${ }^{154} \mathrm{Eu}$ & ${ }^{241} \mathrm{Am}$ \\
\hline 1 & $1 \mathrm{M} \mathrm{HNO}_{3}$, & 15 & 40 & 100 & 100 & 86 & 100 \\
\hline 2 & аналогично № 1 & 8 & 29 & 65 & 67 & - & - \\
\hline 3 & similarly to no. 1 & 6 & 15 & - & 39 & - & - \\
\hline 4 & $8 \mathrm{M} \mathrm{HNO}_{3}$ & 15 & 38 & 100 & 100 & 73 & 65 \\
\hline 5 & аналогично № 4 & 8 & 24 & 41 & 57 & - & - \\
\hline 6 & similarly to no. 4 & 6 & 12 & - & 8 & - & - \\
\hline 7 & $8 \mathrm{M} \mathrm{HNO}_{3}+0,2 \mathrm{M} \mathrm{NaF}$ & 8 & 23 & 37 & 49 & - & - \\
\hline 8 & $\begin{array}{c}\text { аналогично № } 7 \\
\text { similarly to no. } 7\end{array}$ & 6 & 11 & - & 1,4 & - & - \\
\hline
\end{tabular}

Каждый образец в процессе химической и электрохимической дезактивации последовательно обрабатывался в различных режимах, указанных в таблицах. Сравнение результатов проводилось после заключительных обработок.

Загрязнение кобальта на образцах, отобранных с поверхности втулок, в первом случае (электрохимическая дезактивация) снижалось до $23 \%$ (табл. 4, обработка 7). На внутренних образцах керна в первом случае остаточная загрязненность кобальта составляла 11 \% (табл. 4, обработка 8), во втором - 72 \% (табл. 5, опыт 5, № 2).

Остаточная активность ${ }^{134} \mathrm{Cs}$ и ${ }^{137} \mathrm{Cs}$ поверхностных образцов втулок составляла в случае электрохимической дезактивации (37-49) \%, химической дезактивации - до (9-29) \% . После электрохимической обработки внутренних образцов керна остаточная активность ${ }^{137} \mathrm{Cs}$ уменьшалась до $1,4 \%$ (табл. 4, обработка 8), после химической дезактивации - до 31 \% (табл. 5, опыт 5, № 2).

Сравнение полученных данных позволяет считать, что оба способа дезактивации имеют приблизительно одинаковую эффективность, хотя на внутренней поверхности образцов ${ }^{60} \mathrm{Co}$ и ${ }^{137} \mathrm{Cs}$ удалялись интенсивней при электрохимической дезактивации.

Электролиз при высоких плотностях тока порядка 0,2 A/ $\mathrm{cm}^{2}$ приводил к разрушению графитового анода до порошкообразного состояния, что способствовало дополнительной дезактивация образующегося порошка. В качестве электролита использовалась $8 \mathrm{M} \mathrm{HNO}_{3}$, коррозионно не агрессивная по отношению к высоколегированной стали марки 02Х8Н22С6 (ЭП794). Фактически этот электролит может работать длительное время после корректировки, что приводит к образованию минимально возможного объёма жидки РАО.

Таблица 5. Результаты химической дезактивация образцов облученного графита 2, 4 и 9 при $95^{\circ} \mathrm{C}$, продолжительность процесса 2 4

Table 5. Results of chemical decontamination of samples of irradiated graphite 2,4 and 9 at $95^{\circ} \mathrm{C}$, duration is $2 \mathrm{~h}$

\begin{tabular}{|c|c|c|c|c|c|}
\hline \multirow{2}{*}{$\begin{array}{l}\text { Опыт } \\
\text { Exp. }\end{array}$} & \multirow{2}{*}{$\begin{array}{l}\text { Состав раствора } \\
\text { Composition } \\
\text { of solution }\end{array}$} & \multirow{2}{*}{$\begin{array}{l}\text { Oбp. } \\
\text { Spe. }\end{array}$} & \multicolumn{3}{|c|}{$\begin{array}{l}\text { Остаточная загрязненность, \% } \\
\text { Residual contamination, \% }\end{array}$} \\
\hline & & & ${ }^{60} \mathrm{Co}$ & ${ }^{134} \mathrm{CS}$ & ${ }^{137} \mathrm{CS}$ \\
\hline \multirow{3}{*}{1} & \multirow{3}{*}{$8 \mathrm{M} \mathrm{HNO}_{3}$} & 2 & 79 & 25 & 23 \\
\hline & & 4 & 59 & - & 23 \\
\hline & & 9 & 16 & 16 & 9 \\
\hline \multirow{3}{*}{2} & \multirow{3}{*}{$8 \mathrm{M} \mathrm{HNO}_{3}$} & 2 & 80 & 58 & 61 \\
\hline & & 4 & 61 & - & 51 \\
\hline & & 9 & 11 & 12 & 11 \\
\hline \multirow{3}{*}{3} & \multirow{3}{*}{$6 \mathrm{M} \mathrm{HCl}$} & 2 & 81 & 38 & 45 \\
\hline & & 4 & 56 & - & 40 \\
\hline & & 9 & 12 & 15 & 14 \\
\hline \multirow{3}{*}{4} & \multirow{3}{*}{$6 \mathrm{M} \mathrm{HCl}$} & 2 & 77 & 78 & 35 \\
\hline & & 4 & 56 & - & 42 \\
\hline & & 9 & 12 & 13 & 13 \\
\hline \multirow{3}{*}{5} & \multirow{3}{*}{$8 \mathrm{M} \mathrm{HNO}_{3}+0,2 \mathrm{M} \mathrm{HF}$} & 2 & 72 & 52 & 31 \\
\hline & & 4 & 49 & - & 29 \\
\hline & & 9 & 9,6 & 12 & 9 \\
\hline
\end{tabular}

Количество исследуемых добавок в азотную кислоту с целью увеличения ее дезактивирующей способности и скорости разрушения графита ограничилось введением NaF и других кислородосодержащих кислот, не разрушающихся при высоких анодных потенциалах. Введение других добавок, как показали предварительные опыты, приводило к их разрушению под действием подаваемого анодного тока, а как следствие этого - к уменьше- 
нию количества выделяющегося кислорода, и соответственно, - к снижению скорости разрушения графита.

Данные по дезактивации графита при увеличении плотности тока в процессе электрохимического разрушения графитового анода приведены в табл. 6.

Таблица 6. Результаты дезактивации облученного графита в процессе электрохимического разрушения при плотности тока 0,2 A/ $\mathrm{CM}^{2}$

Table 6. Results of decontamination of irradiated graphite at electrochemical destruction at a current density of $0,2 \mathrm{~A} / \mathrm{cm}^{2}$

\begin{tabular}{|c|c|c|c|c|c|c|c|}
\hline \multirow{2}{*}{$\begin{array}{l}\text { Опыт } \\
\text { Exp. }\end{array}$} & \multirow{2}{*}{$\begin{array}{c}\text { Об- } \\
\text { разец } \\
\text { Spe. }\end{array}$} & \multirow{2}{*}{$\begin{array}{l}\text { Состав электролита } \\
\text { Electrolyte composition }\end{array}$} & \multicolumn{5}{|c|}{$\begin{array}{l}\text { Остаточная загрязненность, \% } \\
\text { Residual contamination, \% }\end{array}$} \\
\hline & & & ${ }^{60} \mathrm{Co}$ & ${ }^{134} \mathrm{CS}$ & ${ }^{137} \mathrm{Cs}$ & ${ }^{154} \mathrm{Eu}$ & ${ }^{241} \mathrm{Am}$ \\
\hline 1 & 15 & $8 \mathrm{M} \mathrm{HNO}_{3}$ & 7 & 13 & 14 & 17 & 1 \\
\hline 2 & 7 & $8 \mathrm{M} \mathrm{HNO}_{3}$ & 27 & 6,4 & 10 & - & - \\
\hline 3 & 2 & $8 \mathrm{M} \mathrm{HNO}_{3}+0,1 \mathrm{M} \mathrm{H}_{2} \mathrm{SO}_{4}$ & 22 & 12 & 10 & - & - \\
\hline 4 & 9 & $8 \mathrm{M} \mathrm{HNO}_{3}+0,1 \mathrm{M} \mathrm{H}_{3} \mathrm{PO}_{4}$ & 19 & 7,7 & 9,8 & - & - \\
\hline 5 & 3 & $8 \mathrm{M} \mathrm{HNO}_{3}+0,2 \mathrm{M} \mathrm{NaF}$ & 9,2 & 10 & 5,5 & - & - \\
\hline 6 & 4 & $8 \mathrm{M} \mathrm{HNO}_{3}$ & 42 & - & 14 & - & - \\
\hline 7 & 5 & $8 \mathrm{M} \mathrm{HNO}_{3}+0,2 \mathrm{M} \mathrm{NaF}$ & 26 & - & 0,8 & - & - \\
\hline
\end{tabular}

Из данных, представленных в табл. 6, следует, что введение в азотную кислоту таких добавок, как серная и фосфорная кислоты (до 0,1 M), не приводило к увеличению степени дезактивации порошка графита, полученного при разрушении поверхностных образцов графитовых втулок (опыты 2-4). В присутствии $\mathrm{NaF}$ (опыт 5) остаточная активность ${ }^{137} \mathrm{Cs}$ практически оставалась на том же уровне $(\sim 10 \%)$, при этом остаточная загрязненность по ${ }^{60} \mathrm{Co}$ снижалась по сравнению с азотнокислыми растворами, не содержащими ионов фтора, с 19-27 до $9 \%$, по ${ }^{137} \mathrm{Cs}$ - с 10 до 5,5\% .

При разрушении внутренних образцов из кернов втулок (табл. 6 , опыты 6,7 ) остаточная загрязненность по ${ }^{60} \mathrm{Co} \mathrm{составляла} 42 \%$, а по ${ }^{137} \mathrm{Cs}-14 \%$; ${ }^{134} \mathrm{Cs}$ в таких образцах отсутствовал. Введение в раствор азотной кислоты фторида натрия 0,2 $\mathrm{M}$ по-

\section{СПИСОК ЛИТЕРАТУРЫ}

1. Wood C. Graphite Decommissioning Options for Graphite Treat ment, Recycling, or Disposal, including a discussion of SafetyRelated Issues. - Palo Alto: Electric Power Research Institute, 2006. $-156 \mathrm{p}$.

2. Experimental study on the content and distribution of key nuclides in an irradiated graphite sphere of HTR-10 / H. Li, X. Liu, F. Xie, F. Jia // Nuclear engineering and design. - 2017. V. 323. - P. $39-45$.

3. Kim H.R. The radioactivity estimation of $14 \mathrm{C}$ and $3 \mathrm{H}$ in graphite waste sample of the KRR-2 // Applied Radiation and Isotopes. 2013. - V. 79. - P. 109-113.

4. Actinides in irradiated graphite of RBMK-1500 reactor / R. Plukiene, A. Plukis, V. Barkauskas, A. Gudelis, R. Gvozdaite, G. Duskesas, V. Remeikis // Nuclear Engineering and Design. 2014. - V. 277. - P. 95-105. зволяло снизить остаточную загрязненность по ${ }^{60} \mathrm{Co} \mathrm{до} 26 \%$, а по ${ }^{137} \mathrm{Cs}$ до $0,8 \%$.

В табл. 6 также приведены результаты по дезактивации, полученные при разрушении образца, изготовленного из графитового блока (опыт 1). Остаточная активность ${ }^{60} \mathrm{Co}$ в этом случае снижалась до 7, ${ }^{137} \mathrm{Cs}$ - до $14 \%$. Присутствующий на таких образцах ${ }^{241} \mathrm{Am}$ в основном переходил в раствор азотной кислоты, и его остаточная загрязненность на графите составляла всего $1 \%$, a ${ }^{154} \mathrm{Eu}$ не превышала $17 \%$.

\section{Заключение}

Таким образом, электрохимическая дезактивация графита позволяет снизить количество радионуклидов ${ }^{60} \mathrm{Co}$ в $2-10$ раз и ${ }^{137} \mathrm{Cs}$ в $7-100$ раз. При этом в процессе дезактивации не образуется газообразных продуктов реакции, которые необходимо улавливать, создавая ещё большее количество вторичных РАО.

Значительное снижение мощности поглощенной дозы гамма-излучения и разрушение графита до порошкообразного состояния путем электрохимической обработки без образования при этом радиоактивной пыли позволяет получить отходы графита в компактном виде, удобном для длительного хранения и последующего захоронения. Образующиеся при этом жидкие РА0, представляющие собой соединения азотной кислоты и радионуклидов, могут быть разделены методом дистилляции или путем добавления реагентов с целью осаждения радиоактивных веществ. Это также исключает образование газообразных продуктов реакции, что существенно повышает безопасность известных методов.

Так как способ позволяет существенно снизить концентрацию продуктов деления и трансурановых элементов, то вероятно, исследованный процесс может быть адаптирован для снижения потенциальной опасности графитовых РАО путем удаления наиболее активных долгоживущих радионуклидов ${ }^{36} \mathrm{Cl}$ и ${ }^{14} \mathrm{C}$, находящихся на поверхности. Также возможно снижение класса графитовых РАО, извлеченных из аварийных ячеек.

5. Source term analysis of the irradiated graphite in the core of HTR-10 / X. Liu, X. Huang, F. Xie, F. Jia, X. Feng, H. Li // Science and Technology of Nuclear Installations. - 2017. - P. 1-7.

6. Трехмерное численное моделирование теплового состояния пункта глубинного захоронения радиоактивных отходов в Нижнеканском массиве горных пород / Н.И. Дробышевский, Е.В. Моисеенко, Р.А. Бутов, Ю.Н. Токарев // Радиоактивные отходы. - 2017. - № 1. - С. 65-74.

7. Experience of on-site disposal of production uranium-graphite nuclear reactor / A.0. Pavliuk, S.G. Kotlyarevskiy, E.V. Bespala, E.V. Zakharova, V.M. Ermolaev, A.G. Volkova // Journal of Environmental Radioactivity. - 2018. - V. 184-185. - P. 22-31.

8. Чубреев Д.О., Кузнецов Г.В. Использование глинистых материалов для создания барьера безопасности выводимого из эксплуатации реактора АД // Известия Томского политехнического университета. Инжиниринг георесурсов. - 2016. T. 327. - № 2. - C. 83-87. 
9. Technology and facility for incinerating irradiated reactor graphite / V.A. Kashcheev, A.O. Ustinov, S.A. Yakunin, V.S. Zagumennov, A.0. Pavlyuk, S.G. Kotlyarevskiy, E.V. Bespala // Atomic Energy. - 2017. - V. 122. - № 4. - P. 252-256.

10. Dunzik-Gougar M.L., Smith T.E. Removal of carbon-14 from irradiated graphite // Journal of Nuclear Materials. - 2014. V. 451. - P. 328-335.

11. Li J., Dunzik-Gouga M.L., Wang J. Recent advances in the treatment of irradiated graphite: A review // Annals of Nuclear Energy. -2017 . - V. 110. - P. 140-147.

12. New advances on the thermal behaviour of chlorine in nuclear graphite / A. Blondel, N. Moncoffre, N. Toulhoat, N. Bererd, G. Sibermann, P. Sainsot, J.-N. Rouzaud, D. Deldicque // Carbon. - 2014. - V. 73. - P. 413-420.

13. Thermal treatment of neutron-irradiated nuclear graphite / D. Vulpius, K. Baginski, B. Kraus, B. Thomauske // Nuclear Engineering and Design. - 2013. - V. 265. - P. 294-309.

14. Bespala E., Novoselov I., Ushakov I. Heat transfer during evaporation of cesium from graphite surface in an argon environment // MATEC Web of Conferences. - 2016. - V. 72. - P. 1-5. DOI: $10.1051 /$ matecconf $/ 20167201011$.

15. Heat and mass transfer in cesium evaporation from graphite surface in argon / E.V. Bespala, V.F. Myshkin, A.O. Pavlyuk, I.Yu. Novoselov // Atomic Energy. - 2017. - V. 122. - № 6. P. $400-405$.

16. Decontamination of radioactive concrete / A.Yu. Yurchenko, Yu.V. Karlin, A.N. Nikolaev, 0.K. Karnila, A.S. Barinov // Atomic Energy. - 2009. - V. 106. - № 3. - P. 225-230.

17. Зимон А.Д., Пикалов В.К. Дезактивация. - М.: ИздАТ, 1994. -336 с

18. Головачев М.Г., Лисовских В.Г. Аспекты экологически приемлемой технологии переработки графитовых радиоактивных отходов // Проблемы радиоэкологии и пограничных дисциплин. - 2010. - Вып. 14. - С. 314-326.

19. 0 форме нахождения и характере связи $14 \mathrm{C}$ в облученном графите уран-графитовых ядерных реакторов / Е.В. Беспала, А.О. Павлюк, В.С. Загуменнов, С.Г. Котляревский // Известия вузов. Ядерная энергетика. - 2017. - № 4. - С. 116-126.

20. Modeling the migration of fallout radionuclides to quantify the contemporary transfer of fine particles in Luvisol profiles under different land uses and farming practices / M. Jagercikova, 0. Evrard, J. Balesdent, I. Lefevre, S. Cornu // Soil \& Tillage Research. - 2014. - V. 140. - P. 82-97.
21. Hendry M.J., Wassenaar L.I., Kotzer T. Chloride and chlorine isotopes ( $36 \mathrm{Cl}$ and $837 \mathrm{Cl}$ ) as tracers of solute migration in a thick, clay-rich aquitard system // Water resource research. - 2000. V. 36. - № 1. - P. 285-296.

22. Crooks V.E., Quigley R.M. Saline leachate migration through clay: a comparative laboratory and field investigation // Can. Geotech. J. - 1984. - V. 21. - P. 349-362.

23. Conceptual and numerical models of solute diffusion around a HLW repository in clay / J. Samper, A. Naves, C. Lu, Y. Li, B. Fritz, A. Clement // Physical and Chemistry of the Earth. 2011. - V. 36. - P.1714-1720.

24. Анализ возможности снижения потенциальной опасности графитовых радиоактивных отходов при термической обработке / А.О. Павлюк, С.Г. Котляревский, Е.В. Беспала, А.Г. Волкова, Е.В. Захарова / / Известия Томского политехнического университета. Инжиниринг георесурсов. - 2017. - Т. 328. - № 8. C. $24-32$.

25. Fachinger J., von Lensa W., Podruhzina T. Decontamination of nuclear graphite // Nuclear Engineering and Design. - 2008. V. 238. - P. 3086-3091.

26. Chi S.-H., Kim G.G. Effect of air flow rate on the oxidation of NBG-18 and NBG-25 nuclear graphite // Journal of Nuclear Materials. - 2017. - V. 491. - P. 37-42.

27. Зарецкий С.А., Сучков В.Н., Животинский П.Б. Электрохимическая технология неорганических веществ и химические источники тока. - М.: Высшая школа, 1980. - 423 с.

28. Electrocatalysis and electrode surface properties / A. Frumkin, N. Polianovskaya, I. Bagotskaya, N. Grigoryev // Journal of Electroanalytical Chemistry. - 1971. - V. 33. - № 2. P. $319-328$.

29. A mechanistic model for carbon dioxide corrosion of mild steel in the presence of protective iron carbonate films. P. 1: Theory and verification / M. Nordsveen, S. Nesic, R. Nyborg, A. Stangeland // Corrosion. - 2003. - V. 59. - № 4. - P. 443-456.

30. Немов В.А. Моделирование диффузионно-контролируемого массопереноса в системе электрод-раствор при потенциостатической поляризации межффазной поверхности. Общие и частные краевые условия // Вестник ТОГУ. - 2014. - № 1 (32). C. 27-36.

Поступила 14.04.2018 2.

\section{Информация об авторах}

Павлюк A.O., кандидат физико-математических наук, руководитель группы Научно-исследовательских и опытно-конструкторских работ АО «Опытно-демонстрационный центр вывода из эксплуатации уран-графитовых ядерных реакторов».

Беспала E.B., кандидат физико-математических наук, инженер-физик группы Научно-исследовательских и опытно-конструкторских работ АО «Опытно-демонстрационный центр вывода из эксплуатации уран-графитовых ядерных реакторов» .

Котляревский C.Г., ведущий инженер группы Научно-исследовательских и опытно-конструкторских работ AO «Опытно-демонстрационный центр вывода из эксплуатации уран-графитовых ядерных реакторов».

Волкова A.Г., кандидат химических наук, научный сотрудник лаборатории экологических проблем обращения с радиоактивными и токсичными отходами Института физической химии и электрохимии им. А.Н. Фрумкина Российской академии наук.

Захарова E.B., кандидат химических наук, заведующая лабораторией экологических проблем обращения с радиоактивными и токсичными отходами Института физической химии и электрохимии им. А.Н. Фрумкина Российской академии наук. 


\title{
ELECTROCHEMICAL TREATMENT OF IRRADIATED GRAPHITE OF URANIUM-GRAPHITE NUCLEAR REACTORS
}

\author{
Alexander O. Pavlyuk', \\ seversknet@rambler.ru \\ Evgeny V. Bespala', \\ bespala_evgeny@mail.ru \\ Sergey G. Kotlyarevskiy', \\ skotkyarevkiy@rambler.ru
}

Anna G. Volkova²,

anna.agv@yandex.ru

\section{Elena V. Zakharova²,}

zevchem@rambler.ru

\author{
1 Pilot and Demonstration Center for Uranium-Graphite Nuclear Reactor Decommissioning, \\ 179a, Avtodoroga 13 street, Seversk, 63600, Russia. \\ ${ }^{2}$ A.N. Frumkin Institute of Physical chemistry and Electrochemistry RAS, \\ 31, Leninskiy avenue, Moscow, 119071, Russia.
}

The relevance of the discussed issue is caused by the need in developing the effective methods for treatment of irradiated graphite of uranium-graphite nuclear reactors in order to reduce the potential danger for further burial in geological formations.

The main aim of the study is to develop a method for electrochemical decontamination of graphite radioactive waste in chemically aggressive media and to determine the significant parameters of the process management.

The methods: experimental studies of electrochemical treatment of irradiated graphite in various electrolytes and under various conditions. The results. The principal possibility of electrochemical treatment of graphite radioactive waste is shown in the article. The paper introduces the design of the electrolytic cell for carrying out processing and describes the experimental procedure. The authors have analyzed the electrode processes and identified the factors affecting the electrochemical deactivation efficiency. The dependences of graphite anode destruction rate on the current density and various concentrations of acids are introduced. It was determined that the highest rate of electrode destruction is observed in concentrated hydrochloric acid. The dependence of the removal efficiency of ${ }^{60} \mathrm{CO}^{134+137} \mathrm{CS},{ }^{154} \mathrm{Eu}$, ${ }^{24} \mathrm{Am}$ on composition of the electrolyte was experimentally obtained. It was found that the maximum degree of decontamination of irradiated nuclear graphite at current density of $0,01 \mathrm{~A} / \mathrm{cm}^{2}$ and an electrolyte temperature $(16-20){ }^{\circ} \mathrm{C}$ is achieved in nitric acid with the addition of 0,2 M NaF. The authors carried out the comparative analysis of the experimental results in chemical and electrochemical treatment of graphite radioactive waste. It was shown that electrochemical decontamination of graphite allows reducing the amount of radionuclides ${ }^{60} \mathrm{Co}$ by 2-10 times and ${ }^{137} \mathrm{Cs}$ by 7-100 times. At the same time, at decontamination, no gaseous reaction products that need to be captured, creating a large amount of secondary radioactive waste, occur.

\section{Key words:}

Reactor graphite, electrochemical treatment, radionuclide, uranium-graphite reactor, decontamination.

\section{REFERENCES}

1. Wood C. Graphite Decommissioning Options for Graphite Treat ment, Recycling, or Disposal, including a discussion of Safety-Related Issues. Palo Alto, Electric Power Research Institute Publ., 2006. $156 \mathrm{p}$.

2. Li H., Liu X., Xie F., Jia F. Experimental study on the content and distribution of key nuclides in an irradiated graphite sphere of HTR-10. Nuclear engineering and design, 2017, vol. 323, pp. $39-45$.

3. Kim H.R. The radioactivity estimation of $14 \mathrm{C}$ and $3 \mathrm{H}$ in graphite waste sample of the KRR-2. Applied Radiation and Isotopes, 2013, vol. 79, pp. 109-113.

4. Plukiene R., Plukis A., Barkauskas V., Gudelis A., Gvozdaite R., Duskesas G., Remeikis V. Actinides in irradiated graphite of RBMK-1500 reactor. Nuclear Engineering and Design, 2014, vol. 277 , pp. $95-105$.

5. Liu X., Huang X., Xie F., Jia F., Feng X., Li H. Source term analysis of the irradiated graphite in the core of HTR-10. Science and Technology of Nuclear Installations, 2017, pp. 1-7.
6. Drobyshevskiy N.I., Moiseenko E.V., Butov R.A., Tokarev Yu.N. Three-dimensional numerical modeling of the thermal state of the point of deep burial of radioactive waste in the Nizhnekanskiy massif of rocks. Radioaktivnyye otkhody, 2017, no. 1, pp. 65-74. In Rus.

7. Pavliuk A.O., Kotlyarevskiy S.G., Bespala E.V., Zakharova E.V., Ermolaev V.M., Volkova A.G. Experience of on-site disposal of production uranium-graphite nuclear reactor. Journal of Environmental Radioactivity, 2018, vol. 184-185, pp. 22-31.

8. Chubreev D.0., Kuznetsov G.V. Using clay materials for developing safety barrier of decommissioning AD reactor. Bulletin of the Tomsk Polytechnic University, Geo Assets Engineering, 2016, vol. 327, no. 2, pp. 83-87. In Rus.

9. Kashcheev V.A., Ustinov O.A., Yakunin S.A., Zagumennov V.S., Pavlyuk A.0., Kotlyarevskiy S.G., Bespala E.V. Technology and facility for incinerating irradiated reactor graphite. Atomic Energy, 2017, vol. 122, no. 4, pp. 252-256.

10. Dunzik-Gougar M.L., Smith T.E. Removal of carbon-14 from irradiated graphite. Journal of Nuclear Materials, 2014, vol. 451, pp. 328-335. 
11. Li J., Dunzik-Gouga M.L., Wang J. Recent advances in the treatment of irradiated graphite: A review. Annals of Nuclear Energy, 2017, vol. 110, pp. 140-147.

12. Blondel A., Moncoffre N., Toulhoat N., Bererd N., Sibermann G., Sainsot P., Rouzaud J.-N., Deldicque D. New advances on the thermal behaviour of chlorine in nuclear graphite. Carbon, 2014, vol. 73, pp. 413-420.

13. Vulpius D., Baginski K., Kraus B., Thomauske B. Thermal treatment of neutron-irradiated nuclear graphite. Nuclear Engineering and Design, 2013, vol. 265, pp. 294-309.

14. Bespala E., Novoselov I., Ushakov I. Heat transfer during evaporation of cesium from graphite surface in an argon environment. MATEC Web of Conferences, 2016, vol. 72, pp. 1-5.

15. Bespala E.V., Myshkin V.F., Pavlyuk A.O., Novoselov I.Yu. Heat and mass transfer in cesium evaporation from graphite surface in argon. Atomic Energy, 2017, vol. 122, no. 6, pp. 400-405.

16. Yurchenko A.Yu., Karlin Yu.V., Nikolaev A.N., Karnila O.K., Barinov A.S. Decontamination of radioactive concrete. Atomic Energy, 2009, vol. 106, no. 3, pp. 225-230.

17. Zimon A.D., Pikalov V.K. Dezaktivatsiya [Decontamination]. Moscow, IzdAT Publ., 1994. 336 p.

18. Golovachev M.G., Lisovskikh V.G. Aspects of environmentally acceptable technology for processing graphite radioactive waste. Problemy radioekologii i pogranichnykh distsiplin, 2010, vol. 14, pp. 314-326. In Rus.

19. Bespala E.V., Pavlyuk A.0., Zagumennov V.S., Kotlyarevsky S.G. About chemical form and binding energy of 14c in irradiated graphite of uranium-graphite nuclear reactors. Izvestiya vuzov. Yadernaya energetika, 2017, no. 4, pp. 116-126. In Rus.

20. Jagercikova M., Evrard 0., Balesdent J., Lefevre I., Cornu S. Modeling the migration of fallout radionuclides to quantify the contemporary transfer of fine particles in Luvisol profiles under different land uses and farming practices. Soil \& Tillage Research, 2014, vol. 140, pp. 82-97.

21. Hendry M.J., Wassenaar L.I., Kotzer T. Chloride and chlorine isotopes (36Cl and $\delta 37 \mathrm{Cl}$ ) as tracers of solute migration in a thick, clay-rich aquitard system. Water resource research, 2000, vol. 36 , no. 1, pp. 285-296.
22. Crooks V.E., Quigley R.M. Saline leachate migration through clay: a comparative laboratory and field investigation. Can. Geotech. J, 1984, vol. 21, pp. 349-362.

23. Samper J., Naves A., Lu C., Li Y., Fritz B., Clement A. Conceptual and numerical models of solute diffusion around a HLW repository in clay. Physical and Chemistry of the Earth, 2011, vol. 36, pp. 1714-1720.

24. Pavlyuk A.O., Kotlyarevskiy S.G., Bespala E.V., Volkova A.G., Zakharova E.V. Analysis of capability of reducing potential hazard of radioactive waste under thermal treatment. Bulletin of the Tomsk Polytechnic University, Geo Assets Engineering, 2017, vol. 328 , no. 8, pp. 24-32. In Rus.

25. Fachinger J., von Lensa W., Podruhzina T. Decontamination of nuclear graphite. Nuclear Engineering and Design, 2008, vol. 238, pp. 3086-3091.

26. Chi S.-H., Kim G.G. Effect of air flow rate on the oxidation of NBG-18 and NBG-25 nuclear graphite. Journal of Nuclear Materials, 2017, vol. 491, pp. 37-42.

27. Zaretskiy S.A., Suchkov V.N., Zhivotinskiy P.B. Elektrokhimicheskaya tekhnologiya neorganicheskikh veshchestv $i$ khimicheskie istochniki toka [Electrochemical technology of inorganic substances and chemical sources of current]. Moscow, Vysshaya shkola Publ., 1980. $423 \mathrm{p}$.

28. Frumkin A., Polianovskaya N., Bagotskaya I., Grigoryev N. Electrocatalysis and electrode surface properties. Journal of Electroanalytical Chemistry, 1971, vol. 33, no. 2, pp. 319-328.

29. Nordsveen M., Nesic S., Nyborg R., Stangeland A. A mechanistic model for carbon dioxide corrosion of mild steel in the presence of protective iron carbonate films. P. 1: Theory and verification. Corrosion, 2003, vol. 59, no. 4, pp. 443-456.

30. Nemov V.A. Modeling of diffusion-controlled mass transfer in the electrode-solution system during potentiostatic polarization of the interphase surface. General and private boundary conditions. Vestnik TOGU, 2014, no. 1 (32), pp. 27-36. In Rus.

Received: 14 April 2018.

\section{Information about the authors}

Alexander O. Pavliuk, Cand. Sc., head of the group, Pilot and Demonstration Center for Uranium-Graphite Nuclear Reactor Decommissioning.

Evgeniy V. Bespala, Cand. Sc., physics engineer, Pilot and Demonstration Center for Uranium-Graphite Nuclear Reactor Decommissioning.

Sergey G. Kotlyarevskiy, leading engineer, Pilot and Demonstration Center for Uranium-Graphite Nuclear Reactor Decommissioning.

Anna G. Volkova, Cand. Sc., researcher, A.N. Frumkin Institute of Physical chemistry and Electrochemistry RAS.

Elena V. Zakharova, Cand. Sc., head of the laboratory, A.N. Frumkin Institute of Physical chemistry and Electrochemistry RAS. 